\title{
Original
}

\section{Condiciones de trabajo en el transporte público por carretera}

\section{Working conditions in the public road transport}

\author{
Elena Ordaz Castillo', Jerónimo Maqueda Blasco' \\ 1. Escuela Nacional de Medicina del Trabajo. Instituto de Salud Carlos III. Madrid. España. \\ Recibido: 28-01-14 \\ Aceptado: 20-02-14
}

\section{Correspondencia}

Elena Ordaz Castillo

Correo electrónico: eordaz@isciii.es

Teléfono: 918224025

Resumen

Objetivo: Caracterizar las condiciones de trabajo de los conductores de transporte de viajeros por carretera estableciendo un perfil diferencial con el resto de la población trabajadora.

Métodos: Encuesta dirigida a conductores de transporte de viajeros por carretera para determinar la morbilidad percibida y los factores relacionados con el trabajo que incrementan el riesgo de estos profesionales. En total se entrevistaron 1428 conductores seleccionados por muestreo aleatorio. Se eligió como población de referencia la VI Encuesta Nacional de Condiciones de Trabajo (VI-ENCT/INSHT). Para garantizar la comparabilidad entre nuestro estudio y la VIENCT, se seleccionaron en ambas encuestas varones en edades comprendidas entre los 25 y 65 años. Los cálculos estadísticos se han basado en el $\mathrm{X}^{2}$ para diferencia de proporciones, t-test de diferencia de medias entre variables categóricas y numéricas y estimación del riesgo mediante el OR ajustado por edad.

Resultados: La media de edad de los conductores fue, estadísticamente superior a la población de referencia (diferencia de medias=3,598 [3,05-4,15]).

Los conductores perciben con más riesgo que la población de referencia que el trabajo afecta a su salud $\left(\mathrm{OR}_{\mathrm{aj}}=1,574([1,38-1,79])\right.$. En general, soportan unas cargas globales físicas y mentales más altas que el resto de trabajadores $(\mathrm{p}=0,000)$ y presentan una mayor prevalencia de amenazas de violencia física. Los detalles ergonómicos del puesto de trabajo (espacio, confort del asiento e iluminación) también resultaron estadísticamente peor valorados entre los conductores.

Conclusiones: Se trata por tanto, de un colectivo de trabajadores de más edad que desarrolla sus tareas en un puesto ergonómicamente inadecuado y que soportan una importante carga física y mental, indicadores indispensables a la hora de establecer programas de prevención de accidentes en carretera.

Med Segur Trab (Internet) 2014; 60 (234) 90-98

Palabras clave: Conductores de autobús, transporte de viajeros, condiciones de trabajo.

Abstract

Aims: Characterize the working conditions in public road transport bus drivers and compare them with a control group obtained from the national work force.

Methodology: survey of intercity bus drivers to determine the perceived morbidity and work-related factors that increase the risk of these professionals. A randomized sample of 1428 bus drivers was interviewed 
by means of a questionnaire (personal interview). Our control group was obtained from the VI National Survey of Working Conditions (VI-ENCT/INSHT). The surveys were done in males aged between 25 and 65 to ensure the comparability between our study and VIENCT. Appropriate statistical calculations included: $\mathrm{X}^{2}$ for difference of proportions, t-test of mean difference between categorical and numerical variables and risk estimation using the OR adjusted for age.

Results: The mean age in bus drivers was statistically higher than the reference population.

Bus drivers perceived more frequently than the control group that work conditions affects their health $\left(\mathrm{OR}_{\mathrm{aj}}=1,54([1,36-1,76])\right.$. Likewise, they have higher physical and mental loads than the control group $(\mathrm{p}=0.000)$ and a higher prevalence of threats of physical violence. Ergonomic job details (space, seating comfort and lighting) were also statistically worst rated among drivers.

Conclusions: These results evidence that intercity bus drivers are older than the control group and they have worst ergonomic, physical and mental work-conditions, all of them are essential indicators to establish prevention road accidents programs.

Med Segur Trab (Internet) 2014; 60 (234) 90-98

Keywords: bus drivers, public road transport, working conditions. 


\section{INTRODUCCIÓN}

Las especiales condiciones de trabajo que soportan los conductores de viajeros por carretera guardan una relación muy significativa en la generación de riesgos para la salud del trabajador y para la seguridad de los viajeros y todos los usuarios de la carretera.

El estado de las carreteras y la influencia de factores climáticos se suman a las jornadas de trabajo prolongadas, la desorganización horaria, el apremio del tiempo, el permanente nivel de atención, el trato con los pasajeros o la sobrecarga postural entre otros factores, determinando un perfil de condiciones de trabajo desfavorable frente a otros sectores de actividad.

Según la EPA, de los aproximadamente 1,3 miles de millones de desplazamientos interurbanos que se realizaron en nuestro país durante el año 2012, el 52\% se realizaron en autobús.

La información disponible sobre los riesgos de este colectivo se centra principalmente en estadísticas de siniestralidad laboral: En el año 2012 se registraron 25. 372 accidentes en jornada de trabajo con baja, y 99 partes cerrados de enfermedades profesionales con baja registrados entre conductores de vehículos para el transporte urbano o por carretera según fuentes del Ministerio de empleo y Seguridad Social ${ }^{1}$.

Numerosos estudios identifican una fuerte relación entre los accidentes de conductores de autobuses y/o camiones y somnolencia ${ }^{2,3}$ donde se señalan como principales causas de la falta de sueño (90\%), el consumo de los medicamentos que causan somnolencia (78\%), el consumo de alcohol la noche anterior (23\%), los trastornos respiratorios nocturnos (17\%) y/o las largas horas de conducción ( $>4$ horas).

El estrés relacionado con las condiciones de trabajo es otra de las causas más citadas en el riesgo de sufrir un accidente ${ }^{1,4-6}$. El tiempo al volante, la necesidad de mantener un nivel de atención constante, la dificultad en la conciliación de la vida familiar y laboral, el aislamiento, el trato con los viajeros a menudo relacionado con amenazas físicas o psíquicas, y otros factores relacionados con el trabajo, repercuten en la salud física y psíquica del conductor.

En cuanto a la salud física, y ateniéndonos al cuadro de enfermedades profesionales vigente en nuestro país, el 95\% de las EP declaradas en este colectivo corresponden al grupo de Enfermedades profesionales causadas por agentes físicos. De entre ellas, la enfermedad por fatiga de vainas tendinosas producida por el roce del movimiento repetitivo de los tendones, muñeca y dedos, aglutina casi la totalidad de las enfermedades de este grupo. No obstante, al margen del registro oficial, existen evidencias sobre otras patologías asociadas a las condiciones laborales del conductor que han sido objeto de numerosos estudios. Destacan los trastornos musculo-esqueléticos, principalmente dolor en la parte baja de la espalda ${ }^{7-9}$, dolor en cuello, parte alta de la espalda, hombros y muñecas ${ }^{10,11}$.

El carácter multifactorial de la mayoría de estos trastornos y el papel predominante de factores de confusión relacionados con los hábitos del conductor (dieta inadecuada, tabaquismo, falta de ejercicio físico) hace que sea más difícil establecer asociaciones causales entre la conducción profesional y otros efectos adversos para la salud como las enfermedades, cardiovasculares ${ }^{12,13}$; respiratorias ${ }^{14,15}$, gastrointestinales ${ }^{16}$, estrés y fatiga ${ }^{4 ; 5}$ y enfermedades neoplásicas ${ }^{17,18}$.

En el presente estudio, se pretende identificar los riesgos de la actividad laboral del colectivo de conductores de autobús por carretera y analizar las posibles diferencias respecto al resto de la población trabajadora española. 


\section{MÉTODOS}

Realización de una encuesta dirigida para determinar la morbilidad percibida y los factores de riesgo asociados al trabajo de los conductores de transporte de viajeros por carretera. En total fueron seleccionados 1423 conductores mediante muestreo aleatorio a partir del número total de autorizaciones registradas por la Dirección General de Transporte.

El estudio comparativo de las condiciones de trabajo de los conductores de transporte de viajeros con el resto de la población trabajadora de nuestro país se realizó a través de la VI-Encuesta Nacional de Condiciones de Trabajo del Instituto Nacional de Seguridad e Higiene en el Trabajo (VI-ENCT) ${ }^{19}$. Para garantizar la comparabilidad entre nuestro estudio y la VIENCT, se seleccionó una sub-muestra, de tal manera que el grupo de comparación tuviera una estructura por edad, sexo y situación de alta laboral comparable a la muestra de conductores de nuestro estudio, eliminando de ella a los trabajadores que realizaban su actividad en el sector del transporte viajeros por carretera.

Se compararon distintos indicadores de exposición a riesgos y percepción de las condiciones de trabajo.

La valoración de la carga física y mental se llevó a cabo mediante una serie de preguntas descriptivas de la exposición laboral a este tipo de riesgos. De los indicadores originales con 4 posibles niveles de exposición (siempre o casi siempre, a menudo, a veces, casi nunca o nunca) se generaron dos variables nuevas dicotómicas de carga física y carga mental que respondían a dos categorías: expuestos y no expuestos.

Para la valoración de la carga física global y carga mental global se crearon dos nuevas variables resumen a partir de los indicadores de cada riesgo estableciéndose tres niveles de carga, bajo, medio y alto.

En el análisis estadístico se ha empleado el test del $\mathrm{X}^{2}$ para la diferencia de proporciones; t-test para la diferencia de medias en caso de variables numéricas y categóricas y estimación del riesgo mediante el cálculo del OR ajustado por la variable edad y su Ic95\% (OR ${ }_{\mathrm{aj}}$ IC 95\%]).

\section{RESULTADOS}

La media de edad de los conductores fue de 44,5 $\pm 9,1$ años, estadísticamente superior a la muestra de la población de referencia con una media de 40,9 $\pm 10,01$ años.

En general los conductores perciben con más riesgo que la población de referencia que el trabajo afecta a su salud, una vez ajustado por la variable edad $\left(\mathrm{OR}_{\mathrm{aj}}=1,54[1,36\right.$ $1,76])$.

Figura 1. Prevalencia x 100 trabajadores sobre la opinión de que el trabajo afecta a mi salud

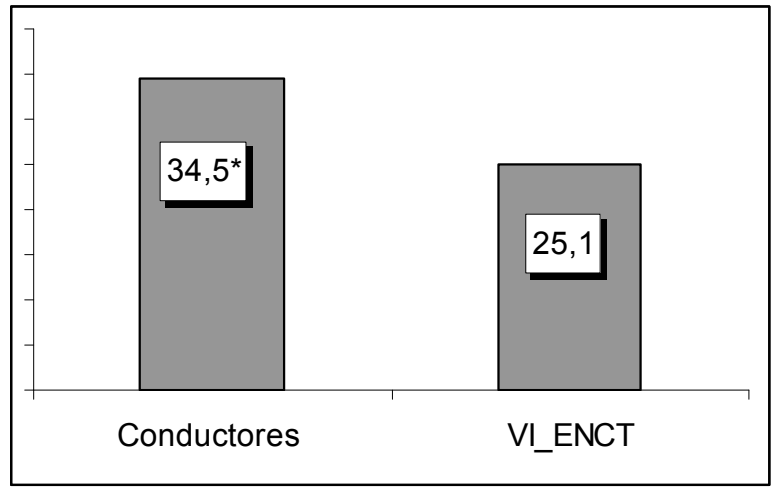

$\mathrm{P}=0,000$ 
En cuanto a la percepción de las condiciones ambientales del puesto de trabajo, los conductores de autobús de viajeros valoran más positivamente factores como la temperatura, el ruido, las vibraciones o la exposición a contaminantes que el resto de la población trabajadora (Tabla I). En esta misma tabla se reflejan también las especiales características del puesto de trabajo de los conductores. En general, trabajan en condiciones de menos espacio ( $6,9 \%$ vs $5,2 \%)$, en asientos más incómodos (3,7\% vs $1,9 \%)$ y con una peor iluminación $(3,7 \%$ vs $2,9 \%)$, diferencias que en todos los casos resultaron estadísticamente significativas.

Tabla I. Prevalencia de exposición a factores ambientales y ergonómicos

\begin{tabular}{lcrc}
\hline \multicolumn{1}{c}{ Indicadores } & Conductores & VI-ENCT & p-value \\
\hline $\mathrm{T}^{\mathrm{a}}$ confortable en verano & $1.188(86,7 \%)$ & $2.743(51,2 \%)$ & 0,000 \\
$\mathrm{~T}{ }^{\mathrm{a}}{ }^{\circ}$ confortable en invierno & $1.291(94,2 \%)$ & $2.883(53,8 \%)$ & 0,000 \\
Ruido muy bajo, casi no hay & $1.019(74,4 \%)$ & $2.881(53,8 \%)$ & 0,000 \\
Vibraciones mano o brazo & $91(6,6 \%)$ & $682(12,7 \%)$ & 0,000 \\
Vibraciones cuerpo & $53(3,9 \%)$ & $454(8,5 \%)$ & 0,000 \\
Contaminantes & $137(10,0 \%)$ & $1.516(28,3 \%)$ & 0,000 \\
Disponer de muy poco espacio siempre o casi siempre & $95(6,9 \%)$ & $277(5,2 \%)$ & 0,000 \\
Disponer de un asiento incómodo & $51(3,7 \%)$ & $101(1,9 \%)$ & 0,000 \\
Iluminación inadecuada & $51(3,7 \%)$ & $153(2,9 \%)$ & 0,000 \\
\hline
\end{tabular}

La distribución de la carga física global es significativamente distinta en ambos grupos como muestra Tabla II, de tal manera que los conductores están expuestos a un trabajo con una exigencia física global "media y alta" superior al conjunto medio del resto de profesiones $(\mathrm{p}=0,000)$.

La probabilidad de estar expuesto a una carga física más alta se multiplica por $2,47[2,20-2,77]$ en los conductores respecto a la población de referencia.

Tabla II. Distribución de la carga física global (OR [IC95\%])

\begin{tabular}{lcrc}
\hline \multicolumn{1}{c}{ Indicadores } & Conductores & \multicolumn{1}{c}{ VIENCT } & ORaj \\
\hline Carga física baja & $229(16,9 \%)$ & $1.931(36,9 \%)$ & \\
Carga física media & $691(50,9 \%)$ & $2.370(45,3 \%)$ & $2,47(2,20-2,77)$ \\
Carga física alta & $438(32,3 \%)$ & $927(17,7 \%)$ & \\
\hline
\end{tabular}

$\mathrm{p}=0,000$

De los cuatro factores de exposición frecuente a carga física, el "Mantenimiento de una misma postura" $\left(\mathrm{OR}_{\mathrm{aj}}=4,51[3,87-5,26]\right)$ junto con la "necesidad de realizar movimientos repetitivos de manos o brazos" $\left(\mathrm{OR}_{\mathrm{aj}}=2,30[2,01-2,63]\right)$ son, sin duda, los factores de carga física más importantes en la actividad del conductor (Tabla III).

Tabla III. Distribución de los indicadores de exposición a carga física en ambas poblaciones

\begin{tabular}{lccc}
\hline \multicolumn{1}{c}{ Indicadores } & Conductores & VIENCT & ORaj \\
\hline Levantar o mover cargas & $342(25,0 \%)$ & $1.270(23,8 \%)$ & $1,11(0,96-1,27)$ \\
Realizar un fuerza importante & $257(18,8 \%)$ & $1.262(23,8 \%)$ & $0,77(0,66-0,89)$ \\
Mantener una misma postura & $1.138(83,6 \%)$ & $2.798(53,0 \%)$ & $4,51(3,87-5,26)$ \\
Realizar movimientos repetitivos de manos o brazo & $1.014(74,3 \%)$ & $2.974(55,9 \%)$ & $2,30(2,01-2,63)$ \\
\hline
\end{tabular}

En cuanto a la carga mental global (Tabla IV), los conductores de autobús soportan una carga mental alta superior a la población de referencia $(p=0,000)$. El análisis de regresión establece que la probabilidad de estar expuesto a una carga mental elevada se multiplica por 3,65[3,22-4,14] en los conductores respecto a la población de referencia. 
Tabla IV. Distribución de la carga mental global (OR $[$ [IC95\%])

\begin{tabular}{lccc}
\hline \multicolumn{1}{c}{ Indicadores } & Conductores & \multicolumn{1}{c}{ VIENCT } & ORaj \\
\hline Carga mental baja & $28(2,1 \%)$ & $405(7,8 \%)$ & \\
Carga mental media & $428(32,5 \%)$ & $2.993(57,6 \%)$ & $3,65(3,22-4,14)$ \\
Carga mental alta & $859(65,3 \%)$ & $1.800(34,6 \%)$ & \\
\hline
\end{tabular}

$$
\mathrm{P}=0,000
$$

De los ocho factores de carga mental contemplados en la encuesta (Tabla V), seis de ellos han resultado más frecuentes en la población de conductores, destacando por encima de todos el tener que "mantener siempre o casi siempre un nivel de atención alto o muy alto" para el desempeño de su trabajo $\left(\mathrm{OR}_{\mathrm{aj}}=10,17\right.$ [7,6-13,5]), el trato con personas ajenas a la empresa $\left(\mathrm{OR}_{\mathrm{aj}} 5,6[4,75-6,61]\right)$ o el uso de nuevas tecnologías $\left(\mathrm{OR}_{\mathrm{aj}} 3,08[2,72\right.$ $3,49])$.

Tabla V. Prevalencia y OR de los indicadores de carga mental

\begin{tabular}{lrcc}
\hline \multicolumn{1}{c}{ INDICADORES (a menudo-siempre) } & \multicolumn{1}{c}{ Conductores } & \multicolumn{1}{c}{ VIENCT } & ORaj \\
\hline Nivel de atención alto o muy alto & $1.314(96,3 \%)$ & $3.848(72,2 \%)$ & $10,17(7,6-13,5)$ \\
Ritmo de trabajo elevado & $741(54,5 \%)$ & $2.338(43,9 \%)$ & $1,63(1,44-1,84)$ \\
Plazos de trabajo estrictos y cortos & $619(45,4 \%)$ & $1.934(36,5 \%)$ & $1,53(1,35-1,73)$ \\
Atender a varias tareas al mismo tiempo & $386(28,5 \%)$ & $2.072(39,0 \%)$ & $0,64(0,56-0,73)$ \\
Trato con personas ajenas a la empresa & $1.177(85,5 \%)$ & $2.820(53 \%)$ & $5,60(4,75-6,61)$ \\
Tareas complejas & $259(19,1 \%)$ & $1.287(24,2 \%)$ & $0,76(0,65-0,88)$ \\
Instrucciones poco claras & $1.066(78,4 \%)$ & $3.655(69,0 \%)$ & $1,62(1,41-1,87)$ \\
Uso de nuevas tecnologías & $746(55,8 \%)$ & $1.559(29,3 \%)$ & $3,08(2,72-3,49)$ \\
\hline
\end{tabular}

Las formas más frecuentes de violencia que sufre el colectivo de conductores son las "Amenazas" con una prevalencia estadísticamente superior a la población de referencia $\left(8,2 \%\right.$ vs $\left.3,9 \% ; O R_{\mathrm{a}} 2,23[1,76-2,83]\right)$. Las conductas de exclusión más frecuentemente señaladas por el colectivo de conductores fueron: nacionalidad $(0,6 \%)$ y edad $(0,4 \%)$, siendo en la sub-muestra de la VI ENCT los principales motivos de discriminación: nacionalidad género $(0,9 \%)$, raza $(0,7 \%)$ y edad $(0,6 \%)$. (Tabla VI).

Tabla VI. Prevalencia indicadores sobre aspectos psicosociales (\%)

\begin{tabular}{lrrrc}
\hline \multicolumn{1}{c}{ Indicadores } & Conductores & \multicolumn{1}{c}{ VI-ENCT } & p-value & ORaj \\
\hline Amenazas de violencia física & $112(8,2 \%)$ & $205(3,9 \%)$ & 0000 & $2,23(1,76-2,83)$ \\
Violencia física por personas de la empresa & $9(0,7 \%)$ & $37(0,7 \%)$ & 1,000 & ---- \\
Violencia física por personas ajenas a empresa & $48(3,5 \%)$ & $214(4,0 \%)$ & 0,434 & ----- \\
Acoso & $7(0,5 \%)$ & $21(0,4 \%)$ & 0,487 & ------ \\
Discriminación por edad & $5(0,4 \%)$ & $32(0,6 \%)$ & 0,412 & ------ \\
Discriminación por nacionalidad & $8(0,6 \%)$ & $49(0,9 \%)$ & 0,319 & ---- \\
Discriminación por género & $2(0,1 \%)$ & $5(0,1 \%)$ & 0,635 & ----- \\
Discriminación por raza/color de piel & $3(0,2 \%)$ & $35(0,6 \%)$ & 0,067 & ----- \\
Discriminación por la orientación sexual & $3(0,2 \%)$ & $4(0,1 \%)$ & 0,148 & ----- \\
\hline
\end{tabular}

\section{DISCUSIÓN}

Los resultados de este estudio revelan que nos encontramos ante un colectivo de trabajadores con una media de edad de 44,5 años, casi cuatro años mayor a la media de la población trabajadora tomada como referencia. Según Navarro \& col (2013), la conducción a partir de los 40 años se relaciona con una perdida en la agudeza visual, que se traduce en pérdidas significativas en la sensibilidad del contraste ante deslumbramientos ${ }^{20}$. 
La percepción sobre la influencia negativa del trabajo en la salud está más arraigada entre los conductores, con una diferencia de aproximadamente 9 puntos porcentajes por encima de la población de referencia y por encima de la media de la UE-27, con un $25 \%$ según la V Encuesta Europea de Condiciones de trabajo ${ }^{21}$.

Se trata por tanto de un colectivo de trabajadores consciente de las especiales condiciones de trabajo que soporta no tanto por las factores ambientales a las que están expuestos (temperatura, ruido o vibraciones), cuyas valoraciones han resultados más positivas que en la población de referencia, si no, principalmente, por la carga física, carga mental y las particularidades del puesto de trabajo, tal y como denota los resultados de este estudio.

El retrato de las condiciones laborales de los conductores se perfila claramente desfavorable respecto a otros colectivos profesionales.

Los conductores tienen una demanda física significativamente mayor $(\mathrm{p}<0,001)$ en lo que se refiere a tener "levantar o mover cargas pesadas," y en lo referido a la carga estática (mantenimiento de postura) y dinámica (movimientos repetidos mano-brazo) que la población trabajadora tomada de referencia. Estos resultados coinciden con los presentados en el estudio salud y condiciones de trabajo en el transporte de mercancías por carretera ${ }^{22}$.

Además el confort del asiento constituye un punto de mejora en el diseño del puesto de trabajo; en líneas generales, disponen de, menos espacio, una iluminación inadecuada y asientos incómodos, tres indicadores relacionados con la aparición de riesgos para la salud y la seguridad tanto por sí mismos como combinados con otros riesgos ya existentes.

El espacio de trabajo del conductor es la piedra angular del trabajo de conducción. Es donde va a estar el trabajador al menos ocho horas sentado, con un alto nivel de atención y concentración, sin posibilidad de tiempos de relajación, descanso y ejercicio físico. En la mayoría de los casos, el lugar de trabajo del conductor no llega al metro cuadrado y en él va situado el asiento, el panel de mandos, el extintor, y los objetos personales del propio conductor, entre otros.

Este espacio debería disponer, al menos, de unas medidas que permitiera el desplazamiento del asiento y del volante para que pudiera adaptarse a las diferentes medidas antropométricas de hombres y mujeres.

La pieza primordial de este espacio de trabajo es el asiento, ya que determina la posición del conductor durante toda la jornada laboral. El sillón debe reunir una serie de características ergonómicas al igual que disponer de una buena suspensión que absorba las vibraciones, dar estabilidad al cuerpo del conductor $\mathrm{y}$, sobretodo, debe poder desplazarse vertical y horizontalmente.

Las desfavorables condiciones ergonómicas, carga física estática, dinámica y un mal diseño del puesto de trabajo repercuten directamente sobre la salud física ${ }^{6-8,10,11}$, del conductor.

Un asiento que no reúna las condiciones necesarias inevitablemente causará enfermedades de tipo músculo-esqueléticas, entre otras lesiones de espalda.

En cuanto a la carga mental, de los ocho indicadores analizados, seis han resultado tener más peso en el colectivo de conductores que en la población de referencia $(\mathrm{p}<0,005)$.

"Mantener un nivel de atención alto", "tratar con personas ajenas a la empresa", "disponer de instrucciones poco claras", "un ritmo de trabajo elevado" y "Trabajar con plazos estrictos" son los principales factores de exposición mental que declaran los conductores a los que hay que sumar el alto grado insatisfacción provocado por la percepción del riesgo de sufrir un accidente, el horario de trabajo, la inestabilidad en el trabajo y las amenazas de violencia física, entre otros, para obtener una visión aproximada del nivel de estrés y fatiga crónica que alcanzan estos trabajadores con las consecuencias que ello produce en la generación de accidentes ${ }^{3-5,23,24}$. 
Se trata por tanto, de un colectivo de trabajadores de más edad, que desarrolla sus tareas en un puesto ergonómicamente inadecuado y que soportan una importante carga física y mental, indicadores indispensables a la hora de establecer programas de prevención de accidentes en carretera.

\section{REFERENCIAS BIBLIOGRÁFICAS}

1. Ministerio de Empleo y Seguridad Social. Anuario de Estadísticas. 2012. Disponible en: http://www. empleo.gob.es/estadisticas/ANUARIO2012/welcome.htm.

2. Leechawengwongs M, Leechawengwongs E, Sukying C, Udomsubpayakul U. Role of drowsy driving in traffic accidents: a questionnaire survey of Thai commercial bus/truck drivers. J Med Assoc Thai 2006 Nov;89(11):1845-50.

3. Philip P. Sleepiness of Occupational Drivers. Industrial Health 2005;43(1):30-3.

4. Kompier MA, Aust B, Van den Berg AM, Siegrist J. Stress prevention in bus drivers: evaluation of 13 natural experiments. J Occup Health Psychol 2000;5(1):11-31.

5. Yamada Y, Mizuno M, Sugiura M, Tanaka S, Mizuno Y, Yanagiya T, et al. Bus drivers' mental conditions and their relation to bus passengers' accidents with a focus on the psychological stress concept. J Hum Ergol (Tokyo) 2008 Jun;37(1):1-11.

6. Jayatilleke AU, Nakahara S, Dharmaratne SD, Jayatilleke AC, Poudel KC, Jimba M. Working conditions of bus drivers in the private sector and bus crashes in Kandy district, Sri Lanka: a case-control study. Inj Prev 2009 Apr;15(2):80-6.

7. Alperovitch-Najenson D, Santo Y, Masharawi Y, Katz-Leurer M, Ushvaev D, Kalichman L. Low back pain among professional bus drivers: ergonomic and occupational-psychosocial risk factors. Isr Med Assoc J 2010 Jan;12(1):26-31.

8. Chen J-C, Chang W-R, Chang W, Christiani D. Occupational factors: associated with low back pain in urban taxi drivers. Occup Med 2005;55:535-40.

9. Hulshof CT, Verbeek JH, Braam ITJ, Bovenzi M, van Dijk FJH. Evaluation of an occupational health intervention programme on whole-body vibration in forklift truck drivers: a controlled trial. Occup Environ Med 2006;64:461-8.

10. Alperovitch-Najenson D, Katz-Leurer M, Santo Y, Golman D, Kalichman L. Upper body quadrant pain in bus drivers. Arch Environ Occup Health 2010 Oct;65(4):218-23.

11. Jensen A, Kaerlev L, Tuchsen F, Hannerz H, Dahl S, Nielsen PS, et al. Locomotor diseases among male long-haul truck drivers and other professional drivers. Int Arch Occup Environ Health 2008 Jul;81(7):8217.

12. Bigert C, Gustavsson P, Hallqvist J, Hogstedt C, Lewne M, Plato N, et al. Myocardial infarction among professional drivers. Epidemiology 2003 May;14(3):333-9.

13. Tuchsen F, Hannerz H, Roepstorff C, Krause N. Stroke among male professional drivers in Denmark, 1994-2003. Occup Environ Med 2006 Jul;63(7):456-60.

14. Ruberto M, Liotti F. [Obstructive sleep apnoea syndrome (OSAS) and mood disorders in a population of public transport drivers]. Med Lav 2011 Mar;102(2):201-7.

15. Hirata RP, Aguiar IC, Nacif SR, Giannasi LC, Leitao Filho FS, Santos IR, et al. Observational study on efficacy of negative expiratory pressure test proposed as screening for obstructive sleep apnea syndrome among commercial interstate bus drivers--protocol study. BMC Pulm Med 2011;11:57.

16. Ronchese F BM. Occupational risks and health disorders in transport drivers. 34(3) 2012;352-9.

17. Murgia N, Dell'Omo M, Gambelunghe A, Folletti I, Muzi G, Abbritti G. Epidemiological evidence of possible musculoskeletal, cardiovascular and neoplastic effects in professional drivers. G Ital Med Lav Ergon 2012;34 (3 Suppl):310-3.

18. Khoubi J, Pourabdian S, Mohebbi I, Tajvidi M, Zaroorian O, Giahi O. Association between the high risk occupations and bladder cancer in Iran: a case-control study. J Occup Med Environ Health 2013; Apr 26(2):205-13

19. Instituto Nacional de Seguridad e Higiene el en Trabajo. VI Encuesta Nacional de Condiciones de Trabajo. http://www.oect.es/Observatorio/Contenidos/InformesPropios/Desarrollados/Ficheros/Informe\%20 VI\%20ENCT\%202007 pdf 2014.

20. Navarro J, Langa A, Ramírez G, Sánchez-Ramos, C. La edad como factor de riesgo en la función visual para la conducción nocturna. Gaceta Optometría 2013;478. 
21. Eurofound. V European Working Condicitons Survey. 2012.

22. Salud y Condiciones de Trabajo en el Transporte de Mercancías por Carretera. 2007.

23. Costa G. Stress of driving: general overview. G Ital Med Lav Ergon 2012;34(3):348-51.

24. Taylor AH DL. Stress, fatigue, health, and risk of road traffic accidents among professional drivers: the contribution of physical inactivity. Annu Rev Public Health 2006;27(371):391.

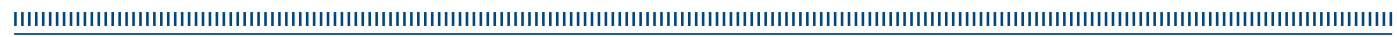

